\title{
Impact of magnetic frustration on the Mott transition within a slave-boson mean-field theory
}

\author{
Imseok Yang, Ekkehard Lange, and Gabriel Kotliar \\ Serin Physics Laboratory, Rutgers University, 136 Frelinghuysen Road, Piscataway, New Jersey 08854, USA
}

\begin{abstract}
We investigate the paramagnetic-metal-to-antiferromagnetic-metal and antiferromagnetic-metal-toantiferromagnetic-insulator transitions using a slave-boson mean-field theory. To this effect, we discuss the ground state of the half-filled Hubbard model as a function of $t^{\prime} / t$ and correlation strength $U$, where $t$ and $t^{\prime}$ are the hopping amplitudes between nearest and next-nearest neighbors, respectively. The metal-insulator transition at a critical $U_{\mathrm{MIT}}$ is of second order for small levels of magnetic frustration, $t^{\prime} / t<0.06$, and of first order for large ones, $t^{\prime} / t>0.06$. The insulator is always antiferromagnetically ordered, while the metal exhibits a second-order transition from a paramagnetic to an antiferromagnetic state up to $t^{\prime} / t=0.14$, as $U$ is increased. We also contrast these findings with what we obtain in Hartree-Fock approximation.
\end{abstract}

PACS Numbers: 75.10.Lp, 71.30.+h, 71.10.Fd, 71.27.+a

The correlation-driven metal-insulator transition, or Mott transition [1], observed in materials such as $\mathrm{V}_{2} \mathrm{O}_{3}$ [2.3] and $\mathrm{NiS}_{2-x} \mathrm{Se}_{x}$ [3] 5 , is a nonperturbative problem usually tackled within the Hubbard model of strongly correlated electrons. This model describes itinerant electrons subject to an on-site repulsion $U$ comparable or greater than the bare bandwidth $2 D$.

In an early work, Brinkman and Rice [6] investigated the Mott transition from the metallic side using Gutzwiller's variational scheme [7,8]. In this approximation, the metal is described as a strongly renormalized Fermi liquid. A low-energy scale $Z D(Z$ is the quasiparticle residue) collapses linearly in $U$ as the Mott transition, occuring at a critical $U_{\mathrm{BR}}$, is approached from the metallic side. $Z D$ is a measure for the renormalized Fermi energy.

We investigate the implications of antiferromagnetic long-range order on either side of the Mott transition. To this effect, we introduce magnetic frustration, which helps stabilize an antiferromagnetic metallic phase for not too large levels of frustration and causes the insulating side to favor antiferromagnetic long-range order [9 12]. We determine the phase diagram and the orders of the transitions in mean-field theory using a slaveboson technique. Our work complements previous studies 110 12 and adds some analytical insights on the interplay of electron-electron correlations and magnetism.

The single-band Hubbard model is given by

$$
\hat{H}=-\sum_{i j \sigma} t_{i j} c_{i \sigma}^{+} c_{j \sigma}+U \sum_{i} \hat{n}_{i \uparrow} \hat{n}_{i \downarrow},
$$

where we take the amplitudes $t_{i j}$ to be nonzero only between nearest and next-nearest neighbors, in which cases they equal $t$ and $t^{\prime}$, respectively. $c_{i \sigma}^{+}$and $c_{i \sigma}$ are creation and annihilation operators for an electron of spin $\sigma$ at site $i$, and $\hat{n}_{i \sigma} \equiv c_{i \sigma}^{+} c_{i \sigma}$. In this work, we consider the two-dimensional cubic lattice and restrict ourselves to half filling and zero temperature.
We use a slave-boson method [13] in which the approximation of Gutzwiller, Brinkman, and Rice is recovered on the saddle-point level, but which at the same time is open to various generalizations such as the inclusion of antiferromagnetic long-range order. The slave-boson method yields a ground-state energy in good agreement with quantum Monte Carlo simulations including antiferromagnetic order [14] and spiral states [15], or with exact diagonalization studies 16]. It has also been used to determine a magnetic phase diagram [17] and to go beyond the Hartree-Fock approximation in problems involving complicated spatial structures such as stripes [18].

In the Kotliar-Ruckenstein approach, two aspects of a physical electron are separated: that it is a fermion and that it affects the occupancy of some site. The first aspect is taken into account by a fermionic field $f_{i \sigma}$, while the possible occupancies of the sites are described by bosonic fields: $e_{i}$ describes empty, $p_{i \sigma}$ singly occupied, and $d_{i}$ doubly occupied sites. The physical electron field is represented as $c_{i \sigma}=\tilde{z}_{i \sigma} f_{i \sigma}$ with $\tilde{z}_{i \sigma}=\left(1-p_{i \sigma}^{+} p_{i \sigma}-\right.$ $\left.d_{i}^{+} d_{i}\right)^{-1 / 2}\left(e_{i}^{+} p_{i \sigma}+p_{i-\sigma}^{+} d_{i}^{+}\right)\left(1-e_{i}^{+} e_{i}-p_{i-\sigma}^{+} p_{i-\sigma}\right)^{-1 / 2}$, while appropriate constraints eliminate unphysical states 13. Thus, the problem posed by the Hubbard interaction is shifted to that of keeping track of the backflow of bosonic excitations, $\tilde{z}_{i \sigma}^{+} \tilde{z}_{j \sigma}$, accompanying the itinerant fermions, $f_{i \sigma}^{+} f_{j \sigma}$.

Proceeding along the lines of Ref. [13], we first set up the functional-integral representation of the Hubbard model in terms of the above-mentioned auxiliary fields, integrate out the fermions, and solve the remaining problem in the saddle-point approximation. To describe antiferromagnetism, we divide the lattice into two sublattices, $A$ and $B$, and look for solutions satisfying the following relations between the sublattice Bose fields: $e_{B}=e_{A}, p_{B \sigma}=p_{A-\sigma}, d_{B}=d_{A}$, and $m=p_{A \uparrow}^{2}-p_{A \downarrow}^{2}$, where $m$ is the staggered magnetization. For our result, we need the dispersion relations of the renormalized quasiparticle bands, 
$\epsilon_{\vec{k} \eta}[X]=-4 t^{\prime} \cos k_{x} \cos k_{y}+\eta t \sqrt{X^{2}+4\left(\cos k_{x}+\cos k_{y}\right)^{2}}$,

where the lattice spacing has been set equal to one, $X$ is some dynamically generated staggered magnetic field, and $\eta= \pm 1$. The equations for the density per site (which at half filling is equal to one) and the staggered magnetization,

$$
\begin{aligned}
1= & \frac{1}{N} \sum_{\vec{k} \eta} f\left(\epsilon_{\vec{k} \eta}[X]-\tilde{\mu}\right), \\
m(X, \tilde{\mu})= & \frac{1}{N} \sum_{\vec{k}} \frac{X}{\sqrt{X^{2}+4\left(\cos k_{x}+\cos k_{y}\right)^{2}}} \\
& \times\left[f\left(\epsilon_{\vec{k}-}[X]-\tilde{\mu}\right)-f\left(\epsilon_{\vec{k}+}[X]-\tilde{\mu}\right)\right],
\end{aligned}
$$

can be solved unambiguously for $X$ and the effective chemical potential $\tilde{\mu}$, to yield functions $\tilde{\mu}(m)$ and $X(m)$. In Eqs. (3) and (4), $N$ is the total number of lattice sites, the sum is over the first Brillouin zone, and $f(\epsilon)=\Theta(-\epsilon)$ is the Fermi function at zero temperature. From the mean-field equation

$$
\frac{\partial q\left(m, d^{2}\right)}{\partial d^{2}} K(m)+U=0,
$$

where the functions $q\left(m, d^{2}\right)$ and $K(m)$ are given by

$$
\begin{aligned}
q\left(m, d^{2}\right)= & \frac{4 d^{2}}{1-m^{2}}\left[1-2 d^{2}+\sqrt{\left(1-2 d^{2}\right)^{2}-m^{2}}\right], \\
K(m) \equiv & \frac{1}{N} \sum_{\vec{k} \eta} \epsilon_{\vec{k} \eta}[X(m)] f\left(\epsilon_{\vec{k} \eta}[X(m)]-\tilde{\mu}(m)\right) \\
& +t m X(m),
\end{aligned}
$$

we obtain the average portion of doubly occupied sites as a function of the staggered magnetization, $d^{2}(m)$. This function along with Eqs. (6) and (7) allow to write the ground-state energy per site as a function of the staggered magnetization as

$$
e(m)=q(m) K(m)+U d^{2}(m) .
$$

This result has an intuitive interpretation: $K(m)$ is the kinetic energy of noninteracting lattice fermions with nearest and next-nearest neighbor hopping, subject to an internal staggered magnetic field $t X(m)$. The renormalization factor $q(m)$ accounts for the reduction of the hopping amplitudes due to the local correlations and is characteristic of the Gutzwiller approximation. In our scheme, $q$ arises from the expectation value $\left\langle\tilde{z}_{i \sigma}^{+} \tilde{z}_{j \sigma}\right\rangle$ and thus represents the average effect of the backflowing slave bosons. The second term in Eq. (8) is the contribution of the Hubbard interaction to the energy.

For $U=0$, Eqs. (5) and (6) yield $d^{2}=\left(1-m^{2}\right) / 4$ and $q=1$, so $\epsilon(m)=K(m)$.
For $t^{\prime}=m=0$, Eqs. (2)-(7) imply: $X=\tilde{\mu}=0 ; K=$ $2 \int_{-\infty}^{0} d \epsilon D_{0}(\epsilon) \epsilon$, where $D_{0}(\epsilon)$ is the density of states for noninteracting electrons; $d^{2}=\frac{1}{4}\left(1-\frac{U}{U_{c}}\right)$ with $U_{c}=8|K|$; and $q=1-\left(\frac{U}{U_{c}}\right)^{2}$. We thus recover a result of Ref. [13].

In the strong-correlation limit, $U \gg t$, we find up to leading order in $t / U: d^{2}=4 t^{2} / U^{2}, q=1-4 t^{2} / U^{2}$, $K=-8 t^{2} / U$, and $m=1-8 t^{2} / U^{2}$. Hence, the groundstate energy is $e=-4 t^{2} / U$. This result is qualitatively correct: In the strong-correlation limit, the halffilled Hubbard model can be mapped onto the Heisenberg antiferromagnet with an exchange coupling constant $J \equiv 4 t^{2} / U$. If we treat the electron spins as Ising spins and use that the coordination number of our twodimensional lattice is four, we also obtain $e=-J$. Therfore, the mean-field theory gives rise to the energy scale $J$ and correctly accounts for a ground-state energy of the order of $-J$ per site. Finally, we note that the leading $1 / U$ corrections do not depend on $t^{\prime}$. More generally, the entire insulating phase is unaffected by next-nearestneighbor hops. $t^{\prime}$ enters only via Eqs. (3), (4) and (7). For the insulator, however, only the band $\epsilon_{\vec{k}-}$ - but the entire one - contributes to the $\vec{k}$ sums in these equations, and $t^{\prime}$ drops out.

The ground state for given model parameters $U$ and $\alpha \equiv t^{\prime} / t$ corresponds to the minimum of the energy function (8). $\alpha$ is a measure for the degree of magnetic frustration and is varied from zero to one. We may restrict ourselves to positive $\alpha$, since a sign change of $\alpha$ is tantamount to a particle-hole transformation, $a_{i \eta} \rightarrow \exp \left\{i \vec{Q} \vec{R}_{i}\right\} a_{i \mid-\eta}^{+}\left(a\right.$ and $a^{+}$describe the quasiparticles with dispersion (2), $\vec{Q}=(\pi, \pi)$, and $\vec{R}_{i}$ is the lattice vector to site $i)$. The evolution of $e(m)$ as a function of $U$ and $\alpha$ reveals how the transitions between the various phases take place.

For $\alpha \neq 0$, The inverse susceptibility, $\chi^{-1}$, is known explicitly and changes its sign from positive to negative as $U$ is increased to above $U_{0} \equiv$ $8|K(0)|(\sqrt{|K(0)| /(|K(0)|-t \gamma)}-1)$. Here, $1 / \gamma \equiv$ $(1 / N) \sum_{\vec{k}}^{\prime} 1 /\left|\cos k_{x}+\cos k_{y}\right|$ and $\sum_{\vec{k}}^{\prime}$ denotes the sum over those regions of the first Brillouin zone which are restricted by the condition $\epsilon_{\vec{k}-}(X=0)<\tilde{\mu}(m=0)<$ $\epsilon_{\vec{k}+}(X=0)$. $U_{0}$ and $\gamma$ depend only on $\alpha$.

Whether the system is metallic or insulating depends on the value of the ground-state magnetization: If it exceeds a certain value, $m_{\mathrm{MIT}}$, a gap opens up in the singleparticle spectrum and the system goes insulating. This can be seen from Eq. (2) if we use that $m$ increases monotonically as a function of $X$. Consequently, the insulator is always antiferromagnetically ordered. We infer from Eqs. (2)-(1) that $m_{\mathrm{MIT}}$ does not depend on $U$, but on the level of magnetic frustration: Due to perfect nesting, $\lim _{\alpha \rightarrow 0} m_{\mathrm{MIT}}=0$. As $\alpha$ is turned on, $m_{\mathrm{MIT}}$ increases monotonically as a function of $\alpha$.

In our numerical investigation, we used a tetrahedron 
method [19] and, for $\alpha<0.2$ and $m<m_{\mathrm{MIT}}$, up to $10^{4} \times$ $10^{4}$ points to do the $\vec{k}$ sums in Eqs. (3), (4) and (4). In the discussion of our numerical results, we must distinguish between three regimes of magnetic frustration and may restrict the discussion of $e(m)$ to positive magnetizations. Fig. 1 illustrates how $e(m)$ evolves as a function of $U$ and $\alpha$. The resulting phase diagram is displayed in Fig. 2 .

For small levels of magnetic frustration, $0<\alpha<0.06$ (first column of Fig. 11), we first find a second-order transition from the paramagnetic to the antiferromagnetic metal at a critical value $U_{\text {mag }}=U_{0}$. Upon further increasing $U$, the resulting minimum is continuously shifted towards higher magnetizations until it crosses $m_{\mathrm{MIT}}$ at a second critical value, $U_{\text {MIT }}$. Consequently, the metalinsulator transition is also of second order. Since $m_{\mathrm{MIT}}$ vanishes as $\alpha \rightarrow 0$, both transitions coincide in this limit, $U_{\text {mag }}=U_{\text {MIT }}=U_{0}$. We also know that $U_{0}=0$ if $\alpha=0$.

For intermediate levels of magnetic frustration, $0.06 \leq$ $\alpha<0.14$ (middle column of Fig. 1), the magnetic transition is still of second order and occurs at $U_{0}, U_{\text {mag }}=U_{0}$. On the other hand, $m_{\mathrm{MIT}}$ is sufficiently large for the metal-insulator transition to take place differently: Before the ground-state magnetization of the antiferromagnetic metal reaches $m_{\text {MIT }}$ upon increasing $U$, a second minimum at a magnetization above $m_{\mathrm{MIT}}$ has emerged and become the absolute minimum of $e(m)$. Consequently, the metal-insulator transition is now of first order. The transition lines $U_{\text {MIT }}(\alpha)$ from the small- $\alpha$ and intermediate- $\alpha$ regimes meet at $\alpha=0.06$ (filled circle in Fig. 2). At this point, the two degenerate minima of the first-order transition merge at $m_{\mathrm{MIT}}$. Finally, the antiferromagnetic metallic phase disappears gradually as $\alpha \rightarrow 0.14$ (filled square in Fig. 2).

For large degrees of magnetic frustration, $\alpha \geq 0.14$ (right column of Fig.11), the antiferromagnetic and metalinsulator transitions coincide, $U_{\mathrm{MIT}}=U_{\mathrm{mag}}$, because the second-order transition at $U_{0}$ is now preempted by the first-order one: By the time the minimum at $m=0$ bifurcates, the one above $m_{\mathrm{MIT}}$ has already evolved into the absolute one, and remains to be so, as $U$ is further increased.

We have also considered the Hubbard model in the Hartree-Fock approximation. Earlier work on the $t-t^{\prime}$ Hubbard model in Hartree-Fock approximation was carried out in Refs. [10], [11], and [20]. As for the twodimensional half-filled case, these works did not conclusively answer whether an antiferromagnetic metal is stable in a certain parameter regime.

Within our formalism, the Hartree-Fock approximation turns out to be tantamount to taking $q=1$ and $d^{2}(m)=\left(1-m^{2}\right) / 4$ in Eq. (8), while the function $K(m)$ is again determined by Eqs. (3), (4), and (7). As a consequence, $\chi^{-1}=2(\gamma t-U / 4)$, which changes its sign at $U_{0}(\alpha)=4 t \gamma(\alpha)$. This is the small- $\alpha$ limit of the corresponding mean-field expression. Expanding
$e^{\mathrm{HF}}(m)$ one step further yields the same $-|m|^{3} \log |m|$ term as in mean-field theory, but with $q=1$. This suggests that the magnetic transition is of second order and takes place at $U_{0}(\alpha)$. In fact, our numerical investigation of the evolution of $e^{\mathrm{HF}}(m)$ as $U$ is increased reveals the following behavior for all finite values of $\alpha$ : After a second-order transition from the paramagnetic to the antiferromagnetic metal at $U_{\text {mag }}=U_{0}(\alpha)$, the minimum of $e^{\mathrm{HF}}(m)$ is rapidly but continuously displaced towards higher magnetizations, until it exceeds $m_{\mathrm{MIT}}$. Thus, the metal-insulator-transition is also of second order. Fig. 3 displays the phase diagram in Hartree-Fock approximation. The error bars of the transition lines $U_{\text {mag }}(\alpha)$ and $U_{\mathrm{MIT}}(\alpha)$ become of the same order than $U_{\mathrm{MIT}}(\alpha)-U_{\mathrm{mag}}(\alpha)$ for $\alpha \leq 0.001$, and our numerics is trustworthy down to about $\alpha \sim 0.002$.

In summary, we have investigated the effect of magnetic frustration on the metal-insulator transition in the two-dimensional half-filled Hubbard model within a slave-boson approach, and we have compared our results to the Hartree-Fock approximation. Within the slaveboson mean-field theory, our main results are: First, magnetic frustration helps stabilize an antiferromagnetic metal for $t^{\prime} / t \leq 0.14$. Second, for $t^{\prime} / t \geq 0.06$, the metalinsulator transition is of first order. Finally, all other transitions between the various phases are of second order. By contrast, in Hartree-Fock approximation, the magnetic and metal-insulator transitions are always separate and of second order. Both in Hartree-Fock approximation and in the slave-boson mean-field theory, the insulator is always antiferromagnetically ordered.

We gratefully acknowledge discussions with R. Frésard and R. Chitra. This work was supported by the NSF DMR 95-29138. E.L. is partly funded by the Deutsche Forschungsgemeinschaft. 

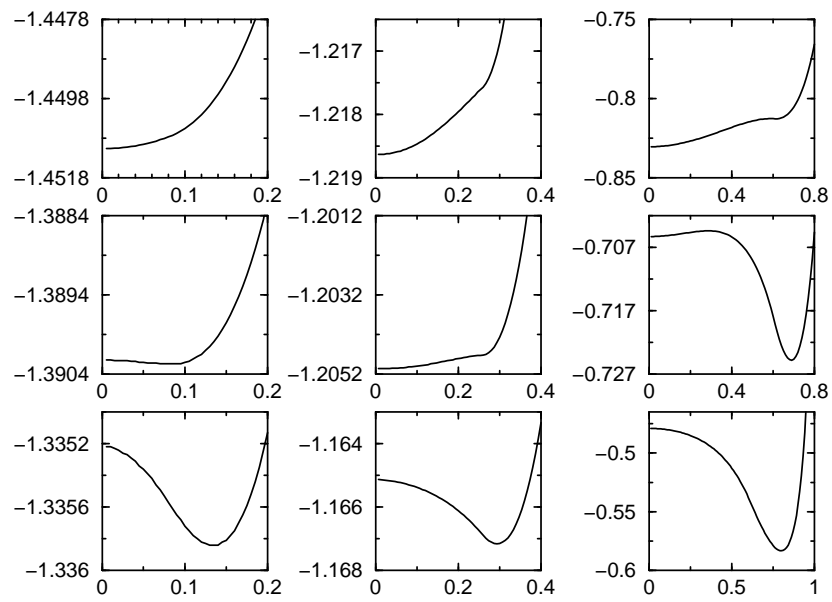

FIG. 1. The function $e(m)$ as a function of $U$ and the magnetic frustration. The columns from left to right correspond to the small-, intermediate-, and large- $\alpha$ regimes, respectively. The respective values are $\alpha=0.02,0.1$, and 0.5 , and correspond to $m_{\mathrm{MIT}}=0.09,0.26$, and 0.61 , respectively. Each column displays how $e(m)$ changes qualitatively upon increasing $U$, from the paramagnetic metal (top row) to the antiferromagnetic insulator (bottom row). The middle row shows examples in the antiferromagnetic metal (first two plots), and one after the metal-insulator transition has taken place but before the local minimum at $m=0$ turns over into a local maximum (plot on the right). The plot in the center has its minimum at a nonzero magnetization, which is not discernible.

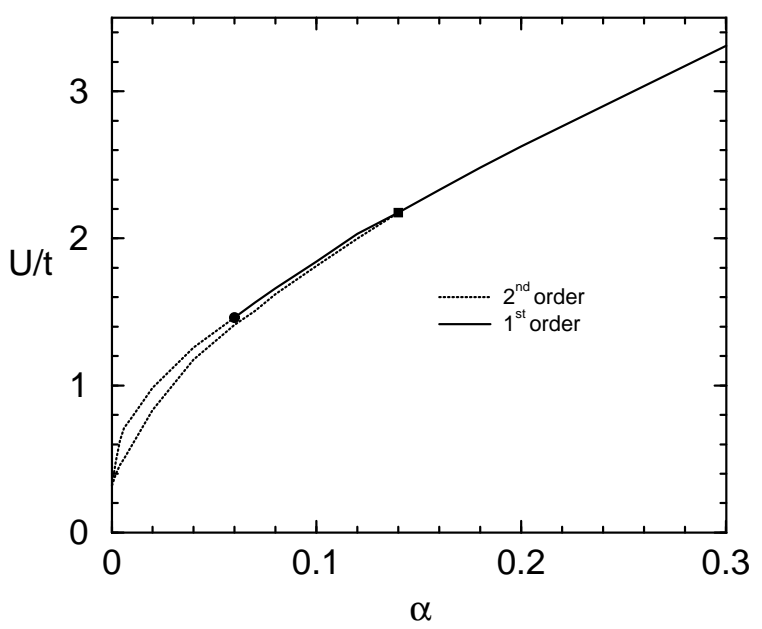

FIG. 2. The various phases and transitions as a function of $\alpha \equiv t^{\prime} / t$ and $U$. The small- $U$ phase is the paramagnetic metal, while the large- $U$ phase is the antiferromagnetic insulator. In between, an antiferromagnetically ordered metallic phase is sandwiched that disappears at a tricritical point (filled square). The dotted and filled lines indicate second- and first-order transitions, respectively. The filled circle marks the point where the metal-insulator transition changes its order.

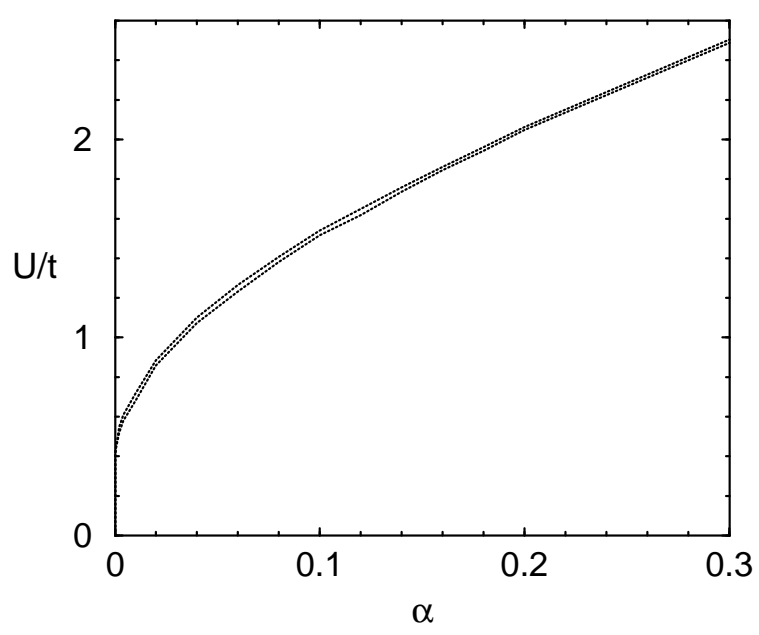

FIG. 3. The phase diagram in Hartree-Fock approximation: The paramagnetic metal (small- $U$ regime) and antiferromagnetic insulator (large- $U$ regime) are separated by a tiny range of $U$ values corresponding to an antiferromagnetically ordered metal. Both transitions are of second order.

[1] N. F. Mott, Philos. Mag. 6, 287 (1961).

[2] S. A. Carter et al., Phys. Rev. B 48, 16841 (1993).

[3] M. Imada, A. Fujimori, and Y. Tokura, Rev. Mod. Phys. 70, 1039 (1998).

[4] F. Gautier et al., Phys. Lett. A 53, 31 (1975).

[5] S. Sudo, J. Magn. Magn. Mater. 114, 57 (1992).

[6] W. F. Brinkman and T. M. Rice, Phys. Rev. B 2, 4302 (1970).

[7] M. C. Gutzwiller, Phys. Rev. Lett. 10, 159 (1963).

[8] M. C. Gutzwiller, Phys. Rev. 137, A1726 (1965).

[9] M. Rozenberg, G. Kotliar, and X. Zhang, Phys. Rev. B 49, 10181 (1994).

[10] H. Lin and J. Hirsch, Phys. Rev. B 35, 3359 (1987).

[11] D. Duffy and A. Moreo, Phys. Rev. B 55, 676 (1997).

[12] R. Chitra and G. Kotliar, cond-mat/9811144.

[13] G. Kotliar and A. E. Ruckenstein, Phys. Rev. Lett. 57, 1362 (1986).

[14] L. Lilly, A. Muramatsu, and W. Hanke, Phys. Rev. Lett. 65, 1379 (1990).

[15] R. Frésard, M. Dzierzawa, and P. Wölfle, Europhys. Lett. 15, 325 (1991).

[16] R. Frésard and P. Wölfle, J. Phys. Condensed Matter 4, 3625 (1992).

[17] B. Möller, K. Doll, and R. Frésard, J. Phys. Condensed Matter 5, 4847 (1993).

[18] S. Seibold and V. Hizhnyakov, Phys. Rev. B 57, 6937 (1998).

[19] G. Gilat and L. J. Raubenheimer, Phys. Rev. 144, 390 (1966).

[20] W. Hofstetter and D. Vollhardt, Ann. Physik 7, 48 (1998). 